\title{
GENERALIZED SUSPENSION THEOREM IN EXTENSION THEORY
}

\author{
LEONARD R. RUBiN \\ University of Oklahoma, USA
}

\begin{abstract}
A. Dranishnikov proved that for each CW-complex $K$ and metrizable compactum $X$ with $X \tau K$, it is true that $(X \times I) \tau(\Sigma K)$. Here, $\Sigma K$ means the suspension of $K$ in the CW-category, and by $X \tau K$ we mean that $K$ is an absolute extensor for $X$. We are going to generalize this result so that $X$ could be either a stratifiable space or a compact Hausdorff space. Since all metrizable spaces are stratifiable, then our result generalizes Dranishnikov's.
\end{abstract}

\section{INTRODUCTION}

In [3], Dranishnikov proved (see Theorem 4) that for each CW-complex $K$ and metrizable compactum $X$ with $X \tau K$, it is true that $(X \times I) \tau(\Sigma K)$, $\Sigma K$ being the suspension of $K$ in the CW-category. By $X \tau K$ we mean that $K$ is an absolute extensor for $X$, or as we prefer to say, $X$ is an absolute co-extensor for $K$. Although Theorem 4 of [3] was stated for an arbitrary metrizable space $X$, the proof that was given requires $X$ to be compact. Here is our main theorem.

THEOREM 1.1. Let $X$ be either a stratifiable or compact Hausdorff space and $K$ be a CW-complex such that $X \tau K$. Then $(X \times I) \tau(\Sigma K)$.

As far as the class of stratifiable spaces is concerned, here are the defining terms.

2010 Mathematics Subject Classification. 54C55, 54C20.

Key words and phrases. Absolute co-extensor, absolute extensor, absolute neighborhood extensor, CW-complex, extension theory, paracompact, shrinking a cover, stratifiable space, stratification, suspension. 
Definition 1.2. A stratification on a space $X$ is a sequence $\left(s_{k}\right)$, such that for each $k, s_{k}$ is a function that assigns to every open set $U \subset X$ an open set $s_{k}(U) \subset X$ such that:

(S1) $\operatorname{cl}_{X}\left(s_{k}(U)\right) \subset U$,

(S2) $\bigcup_{k=1}^{\infty} s_{k}(U)=U$,

(S3) $U \subset V \Rightarrow s_{k}(U) \subset s_{k}(V)$.

A space is called stratified if it has a stratification and is called stratifiable if it is stratified and in addition is a $T_{1}$-space.

This class of spaces was introduced in 1961 by J. G. Ceder [2] under the name $M_{3}$-spaces, and was renamed by C. J. Borges [1] to stratifiable spaces. An exposition of generalized metrizable spaces, including stratifiable spaces, is given by G. Gruenhage [4] in the Handbook of Set-Theoretic Topology. What is important for us is that stratifiable spaces are hereditarily stratifiable, Hausdorff, paracompact, perfectly normal, and that any countable product of stratifiable spaces is stratifiable. They satisfy the subspace theorem in extension theory, namely, if $K$ is a CW-complex, $X$ is a stratifiable space, and $X \tau K$, then for each subspace $Y \subset X, Y \tau K$. This result appears as Theorem 18.12 of [6], but we will only need it in the weak form, i.e., when $Y$ is a closed subspace of $X$. Moreover, every CW-complex is an absolute neighborhood extensor for the class of stratifiable spaces, and every metrizable space is stratifiable. Hence Theorem 1.1 has the following corollary.

Corollary 1.3. Let $X$ be a metrizable space and $K$ a $\mathrm{CW}$-complex such that $X \tau K$. Then $(X \times I) \tau(\Sigma K)$.

\section{Open Sets in Products}

In this presentation, map will always mean continuous function. For each pair $X, Y$ of spaces, let $C(X, Y)$ denote the set of maps of $X$ to $Y$.

Definition 2.1. Let $X$ be a space and $U$ an open subset of $X$. Denote $C(X, U, I)=\{f \in C(X, I) \mid(f(X \backslash U) \subset\{0\}) \wedge(f(U) \subset(0,1])\}$. For each $f \in C(X, U, I)$ and $t \in I$, let $f_{t}^{+}: X \rightarrow \mathbb{R}$ be the map given by $f_{t}^{+}(x)=f(x)+t$ and $f_{t}^{-}: X \rightarrow \mathbb{R}$ the map given by $f_{t}^{-}(x)=-f(x)+t$.

Lemma 2.2. Let $X$ be a space, $U$ an open subset of $X, t \in I$, and $f \in$ $C(X, U, I)$. Then

(1) $\left\{f_{t}^{+}, f_{t}^{-}\right\} \subset C(X, \mathbb{R})$,

(2) for each $x \in U, f_{t}^{-}(x)<f_{t}^{+}(x)$, and

(3) for each $x \in X \backslash U, f_{t}^{+}(x)=t=f_{t}^{-}(x)$.

Definition 2.3. Let $X$ be a space, $U$ an open subset of $X, t \in I$, and $f \in C(X, U, I)$. When we speak of the subspace of $X \times \mathbb{R}$ between $f_{t}^{-}$and $f_{t}^{+}$we refer to $\left\{(x, y) \in U \times \mathbb{R} \mid f_{t}^{-}(x)<y<f_{t}^{+}(x)\right\}$. We shall denote this 
subspace of $X \times \mathbb{R} \operatorname{betw}(f, U, t)$, noting that $\operatorname{betw}(f, U, t) \subset U \times \mathbb{R}$. Let us use $\operatorname{Betw}(X)$ to denote the collection of all such subsets betw $(f, U, t)$ of $X \times \mathbb{R}$.

Whenever $X$ is a space and $f \in C(X, \mathbb{R})$, then by $G_{f}$ we mean the graph of $f$, a closed subset of $X \times \mathbb{R}$. Recall that $G_{f}$ is homeomorphic to $X$. In (2) of the next lemma, the two closed subsets of $X \times \mathbb{R}$ are the respective graphs of $f_{t}^{-}$and $f_{t}^{+}$when these maps are restricted to $\mathrm{cl}_{X} U$.

Lemma 2.4. Let $X$ be a space and $W \in \operatorname{Betw}(X)$. Then

(1) $W$ is an open subset of $X \times \mathbb{R}$, and

(2) in case $W=\operatorname{betw}(f, U, t)$, then $\operatorname{bd}_{X \times \mathbb{R}} W$ equals the union of two closed subsets of $X \times \mathbb{R}$, each of which is homeomorphic to $\mathrm{cl}_{X} U$.

Our interest is in spaces of the form $X \times I$. Lemma 2.4 leads to the next fact.

Lemma 2.5. Let $X$ be a space, $W \in \operatorname{Betw}(X)$, and $M=W \cap(X \times I)$. Then

(1) $M$ is an open subset of $X \times I$, and

(2) $\operatorname{bd}_{X \times I} M$ equals the union of two closed subsets of $X \times I$, each of which is homeomorphic to a closed subspace of $\mathrm{cl}_{X} U$.

Lemma 2.6. Let $Q, U$, be open subsets of a normal space $X$ with $Q \subset$ $\mathrm{cl}_{X} Q \subset U$, and $J$ an interval in $I$. Then there exists an element $W \in \operatorname{Betw} X$ such that $W \cap(X \times I) \subset U \times J$, and $Q \times J \subset W$. Moreover, we may choose $f \in C(X, I)$, an open subset $U_{0}$ of $X$ with $U_{0} \subset U$, and $t \in J$ such that $W=\operatorname{betw}\left(f, U_{0}, t\right)$.

Proof. Let $t$ be the midpoint of $J$, and $\epsilon>0$ equal half the length of $J$. Choose $f \in C(X,[0, \epsilon]) \subset C(X, I) \subset C(X, \mathbb{R})$ so that $f\left(\operatorname{cl}_{X} Q\right) \subset\{\epsilon\}$ and $f(X \backslash U) \subset\{0\}$. Let $U_{0}=\{x \in U \mid f(x)>0\}$. Thus, $U_{0} \subset U$ is an open subset of $X$, and $\operatorname{cl}_{X} Q \subset U_{0}$. One can see that $f \in C\left(X, U_{0}, I\right)$. Put $W=\operatorname{betw}\left(f, U_{0}, t\right)$. We ask the reader, using Definition 2.3, to check that $W$ meets the requirements stated above.

\section{Proof of Main Theorem}

Proof of TheOrem 1.1. We denote the suspension of $K$ as $\Sigma K=$ $\left\{v^{+}, v^{-}\right\} * K$ where $\left\{v^{+}, v^{-}\right\}$is a two-element $\mathrm{CW}$-complex that is disjoint from $K$. Let $A$ be a closed subset of $X \times I$ and $f: A \rightarrow \Sigma K$ a map. Designate $A^{+}=f^{-1}\left(v^{+}\right)$and $A^{-}=f^{-1}\left(v^{-}\right)$. Choose an open cover $\mathcal{E}$ of the regular space $X \times I$ such that if $E \in \mathcal{E}$ and $E \cap A^{+} \neq \emptyset$, then $\left(\operatorname{cl}_{X \times I} E\right) \cap A^{-}=\emptyset$.

Fix $x \in X$. There is a finite open cover $\mathcal{J}_{x}$ of $I$ consisting of intervals, such that for each $J \in \mathcal{J}_{x}$, there exists an element $E_{J} \in \mathcal{E}$ with $\{x\} \times \operatorname{cl}_{I} J \subset E_{J}$. Now choose an open neighborhood $V_{x}$ of $x$ in $X$ having the property that:

$\left(*_{1}\right) V_{x} \times J \subset E_{J}$ for each $J \in \mathcal{J}_{x}$. 
Having done the preceding for each $x \in X$, choose a locally finite open cover $\left\{U_{x} \mid x \in X\right\}$ of $X$ which is a shrinking of $\left\{V_{x} \mid x \in X\right\}$, i.e., for each $x \in X, \operatorname{cl}_{X} U_{x} \subset V_{x}$. Let $\left\{Q_{x} \mid x \in X\right\}$ be an open cover of $X$ that is a shrinking of $\left\{U_{x} \mid x \in X\right\}$. Hence,

$\left(*_{2}\right)$ for each $x \in X, \operatorname{cl}_{X} Q_{x} \subset U_{x} \subset \operatorname{cl}_{X} U_{x} \subset V_{x}$.

Making use of $\left(*_{1}\right)$ and $\left(*_{2}\right)$, we get:

$\left(*_{3}\right)$ for each $x \in X$ and $J \in \mathcal{J}_{x}, U_{x} \times J \subset E_{J}$.

Clearly, $\left\{U_{x} \times I \mid x \in X\right\}$ is a locally finite open cover of $X \times I$. Once again, fix $x \in X$; fix also $J \in \mathcal{J}_{x}$. Apply Lemma 2.6 with $U_{x}, Q_{x}$ respectively in place of $U$ and $Q$, to find $W_{x, J} \in \operatorname{Betw}(X)$ such that,

$\left(*_{4}\right)$ there exist $f \in C(X, I)$, an open subset $U_{0}$ of $X, U_{0} \subset U_{x}$, and $t \in J$ such that $W_{x, J}=\operatorname{betw}\left(f, U_{0}, t\right)$,

$\left(*_{5}\right) M_{x, J}=W_{x, J} \cap(X \times I) \subset U_{x} \times J$, and

$\left(*_{6}\right) Q_{x} \times J \subset W_{x, J}$

Indeed, since $Q_{x} \times J \subset X \times I$, then:

$\left(*_{7}\right) Q_{x} \times J \subset M_{x, J}$

Let $\mathcal{P}_{x}=\left\{M_{x, J} \mid J \in \mathcal{J}_{x}\right\}$. Then $\mathcal{P}_{x}$ is a finite collection of open subsets of $X \times I$ that covers $Q_{x} \times I$, and $\bigcup \mathcal{P}_{x} \subset U_{x} \times I$. Denote $\mathcal{P}=\bigcup\left\{\mathcal{P}_{x} \mid x \in X\right\}$. Then $\mathcal{P}$ is a locally finite open cover of $X \times I$. If we apply $\left(*_{5}\right),\left(*_{2}\right)$, and $\left(*_{1}\right)$, we find that for each $P \in \mathcal{P}$, there is an element $E \in \mathcal{E}$ with $P \subset E$. Therefore, if $P \cap A^{+} \neq \emptyset$, then $\left(\operatorname{cl}_{X \times I} P\right) \cap A^{-}=\emptyset$.

Let $\mathcal{P}^{+}=\left\{P \in \mathcal{P} \mid P \cap A^{+} \neq \emptyset\right\}$. Define $W=\bigcup \mathcal{P}^{+}$. For each $P \in \mathcal{P}^{+}$, $\left(\operatorname{cl}_{X \times I} P\right) \cap A^{-}=\emptyset$. Since $\mathcal{P}^{+}$is a locally finite collection of subsets of $X \times I$, then $A^{+} \subset W \subset \operatorname{cl}_{X \times I} W \subset X \times I \backslash A^{-}$. Again using the fact that $\mathcal{P}^{+}$is a locally finite collection of subsets of $X \times I$, one sees that $\operatorname{bd}_{X \times I} W$ is a closed subset of $\bigcup\left\{\operatorname{bd}_{X \times I} P \mid P \in \mathcal{P}^{+}\right\}$. The latter is a locally finite collection of closed subsets of $X \times I$ each having the form $M_{x, J}$ as in $\left(*_{5}\right)$, so an application of Lemma $2.5(2)$ yields that $\left(\operatorname{bd}_{X \times I} W\right) \tau K$.

Of course, $\operatorname{bd}_{X \times I} W$ is a normal space, so $\left(\operatorname{bd}_{X \times I} W\right) \tau \mathbb{R}$. The space $\Sigma K \backslash\left\{v^{+}, v^{-}\right\}$is homeomorphic to $K \times \mathbb{R}$, so $\left(\operatorname{bd}_{X \times I} W\right) \tau(K \times \mathbb{R})$. Therefore there exists a map $\varphi: \operatorname{bd}_{X \times I} W \rightarrow \Sigma K \backslash\left\{v^{+}, v^{-}\right\} \subset \Sigma K$ that extends the map $f \mid\left(A \cap \operatorname{bd}_{X \times I} W\right)$. Let $\psi=\varphi \cup f:\left(\operatorname{bd}_{X \times I} W\right) \cup A \rightarrow \Sigma K$. Certainly, $\psi$ is a map. Moreover, $\psi\left(\left(A \cap \operatorname{cl}_{X \times I} W\right) \cup \operatorname{bd}_{X \times I} W\right) \subset \Sigma K \backslash v^{-}$. However, $\Sigma K \backslash v^{-}$is an open subset of $\Sigma K$, so it is an absolute neighborhood extensor for both the class of compact Hausdorff spaces and the class of stratifiable spaces. It is also a contractible space. So Theorem 4.7.1 (page 43) of [5] shows that $\left(\operatorname{cl}_{X \times I} W\right) \tau\left(\Sigma K \backslash v^{-}\right)$.

Consider the restriction $\psi^{+}=\psi \mid\left(\left(A \cap \mathrm{cl}_{X \times I} W\right) \cup \mathrm{bd}_{X \times I} W\right)$ with image in $\Sigma K \backslash v^{-}$. There is a map $\xi^{+}: \operatorname{cl}_{X \times I} W \rightarrow \Sigma K \backslash v^{-}$that extends $\psi^{+}$. Similarly, we can find a map $\xi^{-}:(X \times I) \backslash W \rightarrow \Sigma K \backslash v^{+}$that extends 
$\psi^{-}=\psi \mid\left((A \backslash W) \cup \mathrm{bd}_{X \times I} W\right)$ whose image is in $\Sigma K \backslash v^{+}$. The map $\xi^{+} \cup \xi^{-}$: $X \times I \rightarrow \Sigma K$ is an extension of $f$. This ends our proof of Theorem 1.1.

\section{REFERENCES}

1. C. J. R. Borges, On stratifiable spaces, Pacific J. Math. 17 (1966), 1-16.

2. J. G. Ceder, Some generalizations of metric spaces, Pacific J. Math. 11 (1961), 105-125.

3. A. N. Dranishnikov, Extension of maps into $C W$-complexes, Math. USSR-Sb. 74 (1993), 47-56.; Mat. Sb. 182 (1991), 1300-1310 (Russian).

4. G. Gruenhage, Generalized metric spaces, in Handbook of set-theoretic topology, North-Holland, Amsterdam, 1984, 423-501.

5. S. T. Hu, Theory of retracts, Wayne State University Press, Detroit, 1965.

6. I. Ivanšić and L. Rubin, Dimension, extension, and shape, in preparation.

\section{R. Rubin}

Department of Mathematics

University of Oklahoma

Norman, Oklahoma 73019

USA

E-mail: lrubin@ou.edu

Received: 27.8 .2016 . 